\title{
Analítica da Aprendizagem em bases tecnológicas na formação técnica integrada em Informática
}

\author{
Sylvana Karla S. L. Santos ${ }^{1}$, Kadidja Valéria R. Oliveira ${ }^{1}$ \\ ${ }^{1}$ Instituto Federal de Brasília \\ \{sylkarla,kadidja.oliveira\}@gmail.com
}

\begin{abstract}
The scenario of changes in on-site teaching institutions, caused by the impacts of the COVID-19 pandemic, boosted the use of digital information and communication technologies to resume classes. The active methodologies were strategically implemented for remote education. This study presents an experience report on the use of Learning Analytics to collect, process and give visibility to the results achieved in carrying out an Integrated Project of computing disciplines. It concludes that it was possible to develop the technological bases, foster the ability to work collaboratively, identify individual skills and enhance the role of students.
\end{abstract}

Resumo. O cenário de mudanças nas instituições de ensino presenciais, ocasionado pelos impactos da pandemia da COVID-19, impulsionou o uso das tecnologias digitais de informação e comunicação para a retomada das aulas. As metodologias ativas foram implementadas de forma estratégica para o ensino remoto. Este estudo apresenta um relato de experiência no uso da Analítica da Aprendizagem para coletar, processar e dar visibilidade aos resultados alcançados na realização de um Projeto Integrado de disciplinas de computação. Conclui que foi possível desenvolver as bases tecnológicas, fomentar a capacidade de trabalhar de forma colaborativa, identificar competências individuais e potencializar o protagonismo dos discentes.

\section{Introdução}

As mudanças sociais, culturais e educacionais geradas pela pandemia da COVID- $19^{1}$ e o fechamento das escolas no Brasil e em outros países motivou um reconhecimento de que as escolas desempenham um papel essencial além da aprendizagem [Fullan et al. 2020]. A suspensão das atividades letivas e o retorno tardio das aulas, de forma remota, mobilizou a comunidade acadêmica do Instituto Federal de Brasília (IFB) para um planejamento estratégico ao processo de ensino-aprendizagem, que considerasse o total distanciamento físico e o uso de tecnologias digitais de informação e comunicação e metodologias ativas nos processos comunicacionais e na continuidade das atividades letivas de forma satisfatória.

Nesse contexto, esse estudo enfatiza, a partir da concepção e realização de um Projeto Integrado de disciplinas de computação ${ }^{2}$, o engajamento de docentes e discentes

\footnotetext{
${ }^{1}$ A COVID-19 é uma doença causada pelo novo coronavírus, denominado SARS-CoV-2, que apresenta um espectro clínico variando de infecções assintomáticas a quadros graves. Para saber mais: https://coronavirus.saude.gov.br/sobre-a-doenca. Acesso em 08 abr. 2021.

2 O Curso Técnico de Informática Integrado ao Ensino Médio obedece ao disposto na Lei de Diretrizes e Bases da Educação Nacional - LDB, n 9.394/96, ao Decreto $n^{\circ}$ 5.154/04, que regulamenta os dispositivos
} 
para o desenvolvimento tecnológico, a metodologia de projetos, a habilidade de trabalhar em grupo e a articulação e aplicação dos conteúdos apreendidos. Tem como objetivo a análise dos resultados de aprendizagem, considerando como indicadores as bases tecnológicas de componentes curriculares do Projeto Pedagógico do Curso de Ensino Médio Integrado (EMI) em Informática, no tocante aos impactos causados pelas mudanças no contexto educacional, com o formato do ensino remoto.

Diante disso, foi adotada a metodologia de Aprendizagem Baseada em Projetos, com uma proposta de desenvolvimento de um projeto de Sistema de Informação temático e relacionado à área fim da formação dos discentes ${ }^{3}$. Considera as fases de desenvolvimento de sistemas da Engenharia de Software, como a concepção, análise de requisitos, e o desenvolvimento da documentação, a modelagem do Banco de Dados, a definição do Sistema Operacional e culmina com a construção de um Protótipo, agregado à documentação do sistema, como produto do projeto.

Assim, são relatadas as etapas de desenvolvimento do Projeto Integrado junto às turmas do segundo ano do curso EMI em Informática do IFB, com o propósito de identificar, pela ótica da Analítica de Aprendizagem ${ }^{4}$, a inclusão dos estudantes no processo de aprendizagem ativa e colaborativa, e no incentivo ao protagonismo dos discentes na realização de atividades remotas, em tempos de distanciamento físico, mas não social.

Após essa breve contextualização, a seção 2 traz uma revisão de literatura que aborda os conceitos de Aprendizagem Baseada em Projeto e da Analítica da Aprendizagem. A seção 3 descreve a metodologia adotada, seguida da seção 4 que indica os resultados com discussões e, por fim, são apresentadas as considerações na seção 5.

\section{Revisão de Literatura}

\subsection{Aprendizagem Baseada em Projetos}

A Aprendizagem Baseada em Projetos (ABP) ou Problem Based Learning (PBL) é um modelo de ensino que viabiliza aos estudantes lidar com os obstáculos e os questionamentos do mundo atual, considerados de interesse para serem abordados e solucionados [Almeida et al. 2017].

Trata-se de uma metodologia ativa que possibilita a participação discente, de forma individual ou em equipe, para trabalhar habilidades, como pensamento crítico e criativo, e a percepção de que, para realizar uma tarefa, existe mais de uma forma. A avaliação ocorre tanto com base no desempenho ao longo do processo quanto com a entrega do produto final [Moran 2018].

\subsection{Analítica de Aprendizagem}

O termo Analítica da Aprendizagem (Learning Analytics LA) é entendido como a mediação, coleta, análise e o reporte de dados sobre os estudantes e seus contextos, com o propósito de entender a aprendizagem e os ambientes em que ocorre [SoLAR 2011].

referentes à Educação Profissional previstos na LDB, e sua articulação com o ensino médio, na forma integrada. O plano de Curso está disponível em: < fonte omitida para avaliação> Acesso em 06 abr. 2021.

3 Área do curso: Informática.

${ }^{4}$ Uma das aplicações do Data Analytics, pode ser o Educational Analytics, no campo da Educação. 
Embora considerada interdisciplinar, a área de mineração de dados educacionais está mais relacionada a pesquisas desenvolvidas na área de Computação [Nunes et al. 2015].

Utiliza-se a técnica de Analítica da Aprendizagem para o propósito de compreender o progresso dos estudantes ao longo do desenvolvimento do Projeto Integrado, proposto para a $\mathrm{ABP}$, tendo como identificadores as habilidades $\mathrm{e}$ as competências alcançadas pelo engajamento dos discentes no produto final.

Almeja-se, por meio dos dados coletados e visualizados, compreender os insights propiciados pela LA [Filatro 2021], no sentido de contribuir com melhorias para a realização de novos Projetos Integrados, de tal forma que sejam incluídas disciplinas do eixo da informação e comunicação, integradas com as disciplinas elencadas no ensino médio e que potencialize o processo de intervenções educacionais.

Em razão disso, com o uso de LA é possível identificar, por exemplo, os padrões comportamentais e sociais que impactam no aprendizado do estudante [Brasil et al. 2018].

\section{Metodologia}

A pesquisa possui abordagem qualitativa e quantitativa, cujo objetivo é descrever a experiência de duas docentes durante a condução de turmas do curso de EMI em Informática do IFB, desenvolvida em 2020. A matriz curricular do curso é composta por 12 componentes da formação geral do curso e seis da formação profissional [IFB 2014]. O Projeto Integrado alcançou três dos componentes curriculares técnicos, sendo: Engenharia de Software, Banco de Dados e Sistemas Operacionais.

Por se tratar de um curso técnico integrado, o planejamento do projeto considerou reunir o conhecimento adquirido pelos discentes, ao longo do segundo semestre letivo, para evidenciar a prática do aprendizado dos alunos e aplicá-la na construção da documentação de um projeto de software. Este projeto foi dividido em fases, com base na Metodologia EduScrum ${ }^{5}$, para auxiliar no trabalho desenvolvido em grupos de estudantes. Considerando uma atividade que estava em andamento no primeiro semestre letivo, foi proposta a sua continuidade para a criação de um sistema de perguntas e respostas, comumente denominado Quiz [Petri et al. 2016].

Para auxiliar na elaboração do documento do sistema, as docentes forneceram um modelo (template) composto por uma estrutura com itens básicos da documentação, como propósito do documento, escopo do produto, requisitos funcionais e não funcionais, diagrama de caso de uso, modelagem do banco de dados e projeto de interface (protótipo de baixa fidelidade). A divisão dos estudantes em grupos, também denominados 'times', foi uma premissa para a realização do projeto, o que permitiu identificar as habilidades de cada membro da equipe e viabilizar a divisão de papéis na equipe de desenvolvimento. A divisão em times se deu pela escolha dos estudantes.

O ciclo de vida do projeto foi baseado na metodologia ABP, descrita anteriormente, e estruturado em três etapas, sendo: (1) criação e planejamento: teve início com a realização de uma oficina de planejamento que contou com a participação dos docentes e discentes das turmas do $2^{\circ}$ ano do curso, onde foi apresentado o modelo Canvas de Projeto [Finochio 2020] para ser preenchido, coletivamente, de acordo com a definição dos temas de cada grupo; (2) desenvolvimento: a partir da elaboração de um cronograma,

\footnotetext{
5 eduScrum - https://www.eduscrum.com.br/
} 
cada grupo identificou as entregas (sprint) a serem feitas. O registro das entregas foi feito no ambiente virtual de aprendizagem Moodle, adotado pelo colegiado do curso. O acompanhamento dos grupos foi realizado durante encontros coletivos, conduzidos pelas duas docentes e com duração de até uma hora semanal. Os encontros eram gravados e disponibilizados aos estudantes que não conseguiam estar presentes de forma síncrona; (3) monitoramento e avaliação: diante da ausência de alguns membros dos grupos e das dúvidas recorrentes que surgiram no decorrer do desenvolvimento do projeto, as docentes definiram a realização de momentos síncronos com cada grupo. Esse novo formato contribuiu para identificar falhas pontuais e esclarecer dúvidas mais específicas; (4) registros: a realização de encontros síncronos por equipe ajudou a aumentar a participação e o engajamento dos estudantes. Para contribuir posteriormente com a continuidade da discussão, as docentes disponibilizaram um fórum virtual como um canal de comunicação assíncrona para que os times pudessem registrar dúvidas que surgissem e obter respostas com mais brevidade.

O universo dos participantes da pesquisa é composto por 63 estudantes, considerando as duas turmas do curso e todos os discentes matriculados no $2^{\circ}$ ano em 2020. Desse total, identificou-se aqueles que evadiram após o retorno remoto, os que não aderiram ao ensino remoto, estando ausentes das atividades assíncronas e dos encontros síncronos, e aqueles que não participaram do Projeto Integrado, totalizando 44 estudantes frequentes. Ao final, a amostra alcançada foi de 29 respondentes, o que corresponde a $66 \%$ da turma. As perguntas foram divididas em três grupos com o objetivo, principalmente, de verificar a aprendizagem na perspectiva do estudante em cada uma das habilidades necessárias a serem alcançadas e descritas no Plano de Curso [IFB 2014].

Considerando o tempo como um fator crítico para o término do ano letivo e das atividades do projeto, foram definidas entregas (sprints) parciais e finais, de acordo com cada componente curricular, segundo o quadro 1:

Quadro 1. Componente curricular e sprint definida.

\begin{tabular}{|l|l|}
\hline Componente curricular & Sprint \\
\hline Engenharia de Software & $\begin{array}{l}\text { O projeto deve possuir funcionalidades (requisitos) descritas e validadas; } \\
\text { Para o levantamento de requisitos, deverá apresentar uma técnica de } \\
\text { levantamento de requisitos aplicada. } \\
\text { Modelagem (UML) dos Diagramas de Caso de uso, Classe, Atividade e } \\
\text { Sequência. }\end{array}$ \\
\hline Banco de Dados & $\begin{array}{l}\text { Apresentar a Modelagem Diagrama Entidade-Relacionamento } \\
\text { (cardinalidade e normalização) dos dados } \\
\text { Construção do Dicionário de Dados } \\
\text { CRUD }\end{array}$ \\
\hline Sistemas Operacionais & $\begin{array}{l}\text { Identificar o sistema operacional da aplicação. } \\
\text { Indicar as vantagens e desvantagens para a escolha do sistema operacional. }\end{array}$ \\
\hline
\end{tabular}

Fonte: elaborado pelas autoras, 2020.

A seguir, são apresentados os dados coletados e os resultados alcançados.

\section{Resultados e Discussões}

A autoavaliação dos discentes quanto à participação no Projeto Integrado foi realizada a partir de um formulário online, disponibilizado para receber as respostas durante 10 dias 
no mês de março/2021. A adesão alcançou 29 respondentes, com maioria proporcional de estudantes da turma $2 \mathrm{~A}$, conforme mostra a figura 1 .

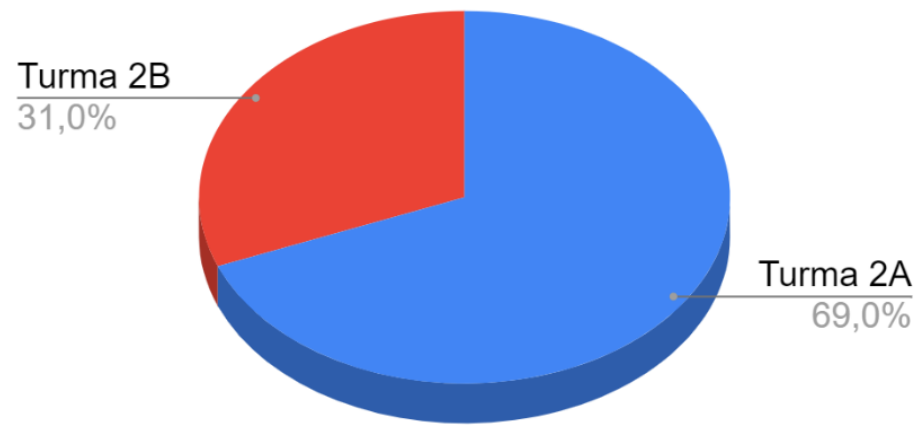

Figura 1. Quantidade de estudantes respondentes por turma.

Fonte: dados coletados pelas autoras, 2021.

Nas seções de perguntas subsequentes, foram abordadas as habilidades alcançadas em cada componente curricular. A escala de avaliação adotada permitiu ao estudante pontuar entre 10 e 0 , sendo 10 caso a habilidade tenha sido alcançada totalmente e 0 para indicar que a habilidade não foi alcançada, destacada no eixo x do gráfico. A quantidade de respondentes está descrita no eixo y. Para a análise do aproveitamento dos estudantes, foi adotada a divisão da pontuação em três intervalos na escala 10 a 0 , sendo: 10 a 8 muito satisfatório, 7 a 5 - satisfatório e 4 a 0 - insatisfatório.

A primeira análise diz respeito ao componente curricular Engenharia de Software em relação aos conceitos: (1) introdução aos conceitos da disciplina; (2) diagramas da UML; (3) projeto de software; (4) requisitos de software e (5) descrição de casos de uso, conforme mostra a figura 2 .
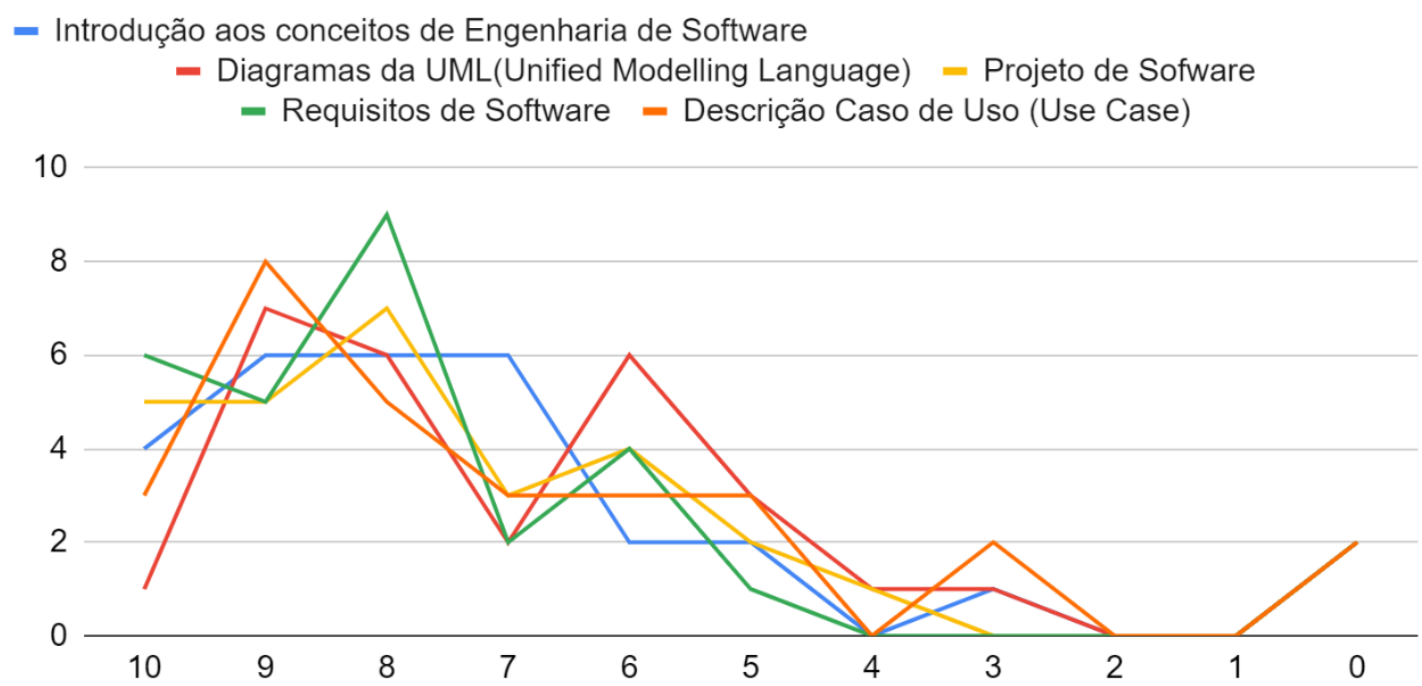

Figura 2. Engenharia de Software.

Fonte: dados coletados pelas autoras, 2021.

Percebe-se que o item 1, que trata da introdução aos conceitos de engenharia de software, foi avaliado com indicador muito satisfatório pela maioria $(55,2 \%)$, o que demonstra que houve uma boa compreensão dos conceitos pelos estudantes, assim como o item 2, que aborda os diagramas da UML, que atingiu 48,3\%. Os demais itens 
alcançaram 58,6\%, $69 \%$ e 55,2\%, respectivamente, sendo considerados muito satisfatórios.

A análise do componente curricular Banco de Dados (figura 3) pontuou com indicadores os itens: (1) ferramentas de edição de diagramas, com 65,5\%; (2) modelo lógico do banco de dados, com 62,1\%; (3) modelo físico do banco de dados, atingindo 69\%; (4) dicionário de dados, com 62,1\% e, finalmente, (4) CRUD, acrônimo de CreateRead-Update-Delete, com 55,2\%. Estes indicadores sinalizam que os conceitos tiveram compreensão muito satisfatória para a maioria dos respondentes.

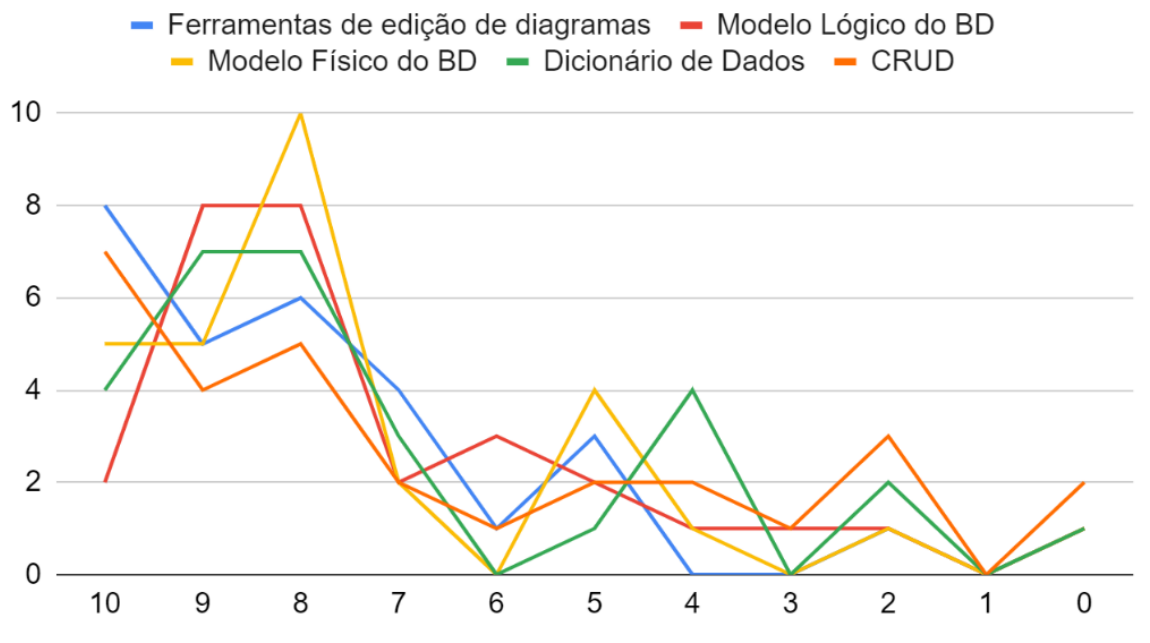

Figura 3. Banco de Dados.

Fonte: dados coletados pelas autoras, 2021.

O terceiro e último item a ser avaliado estava associado ao componente Sistemas Operacionais (SO) que buscou identificar: (1) conceitos básicos (71,4\%); (2) justificativa para escolha do SO para o projeto, com 64,3\%; (3 e 4) vantagens e desvantagens de um SO, com $67,9 \%$ cada, todos considerados muito satisfatórios pelos respondentes, conforme indica a figura 4. Esses índices, superiores a $60 \%$, indicam que houve uma facilidade maior por parte dos estudantes em associar os conceitos à prática no desenvolvimento do projeto.

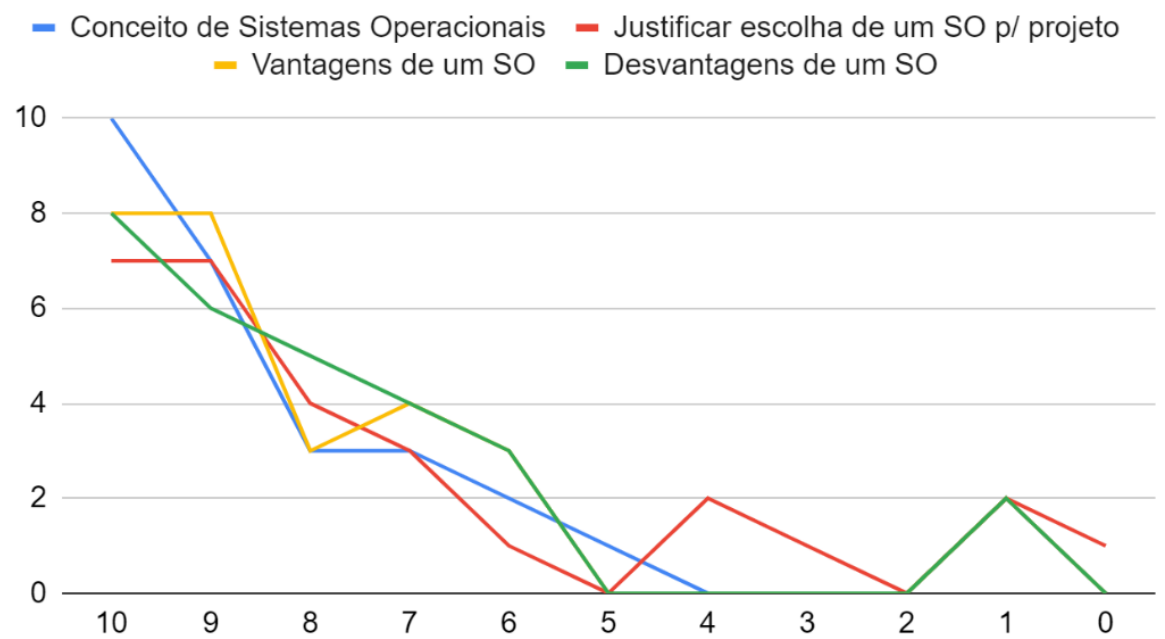

Figura 4. Sistemas Operacionais.

Fonte: dados coletados pelas autoras, 2021. 
De forma geral, os resultados positivos são percebidos pela representação gráfica de cada figura, de acordo com uma maior frequência à esquerda, onde a quantidade de respostas corresponde a um maior índice de avaliação, e decresce com menor frequência à medida que se aproxima dos menores índices de avaliação que estão à direita.

Além das questões objetivas, o instrumento de coleta considerou um campo aberto para que os respondentes pudessem complementar com comentários, caso desejassem. A maioria das opiniões foi positiva quanto à satisfação com a realização do projeto de forma integrada, a prática da integração e o trabalho em grupo, favorecendo um resultado significativo.

\section{Considerações}

Este relato de experiência buscou descrever o planejamento e execução de um Projeto Integrado em turmas do segundo ano do ensino médio integrado ao curso técnico em Informática, utilizando a metodologia da Aprendizagem Baseada em Projetos.

Ao questionar diretamente os estudantes, por meio de um instrumento de coleta de dados online ${ }^{6}$, buscou-se compreender como ocorreu a evolução de aprendizagem - $a$ aplicação dos conhecimentos apreendidos em disciplinas no semestre anterior - no decorrer do desenvolvimento do Projeto Integrado, considerando o alcance de habilidades e competências dos discentes na entrega do produto final com o auxílio da técnica Analítica de Aprendizagem.

Nesse contexto, os insights buscaram identificar quando os estudantes estavam prontos para avançar para o próximo estudo, quando um estudante estaria em risco de não concluir o componente curricular, qual o melhor caminho para um estudante seguir num percurso de aprendizagem determinado, entre outras questões que podem ser respondidas no uso dessa metodologia pautada na mineração de dados.

A partir da construção coletiva do documento do sistema, desenvolvido em times formados pelos discentes, foi possível promover habilidades técnicas, associadas às três componentes curriculares do curso de forma integrada, bem como fomentar a capacidade de trabalhar de forma colaborativa, identificar competências individuais para dividir tarefas e potencializar o protagonismo dos discentes.

As dificuldades pontuadas por alguns estudantes podem indicar a ausência de alcance de competências específicas e necessárias, considerando a deficiência de interação entre determinados membros dos grupos. Diante do atual cenário de ensino remoto, as docentes têm consciência de que não foi possível a todos atingirem o sucesso desejado. No entanto, esse resultado serve como ponto de controle para que a execução do Projeto Integrado possa ser melhorado em outras ofertas, ainda que mediado pelas Tecnologias Digitais de Informação e Comunicação e pelas metodologias ativas.

\section{Referências}

Almeida J., Nunes M., Almeida F. (2017) "Desenvolvimento de jogos com uso de Pensamento Computacional e Aprendizagem Baseada em Projetos", Encontro Nacional de Computação dos Institutos Federais, 4., p. 252-255. DOI: https://doi.org/10.5753/encompif.2017.9937

\footnotetext{
6 Para que os estudantes tivessem maior liberdade de participar da pesquisa, não foi solicitada a identificação do respondente.
} 
Brasil, P. C., Medeiros, T. J., Nunes, I. D. (2018) "O uso de Learning Analytics no Brasil: uma revisão sistemática da literatura", Revista Tecnologias na Educação, 10(26), p. 95-112. Disponível em: https://tecedu.pro.br/ano10-numerovol26-edicao-tematicaviii/ Acesso em 06 abr. 2021.

Filatro, A. (2021) "Data Science na educação presencial, a distância e corporativa". São Paulo: Saraiva Educação, 224p.

Finocchio Jr, J. (2020) "Project Model Canvas", 2. ed. São Paulo: Saraiva Educação. $232 p$.

Fullan, M., Quinn, J., Drummy, M., Gardner, M. (2020), "Educação reinventada: O futuro da aprendizagem". Um documento de posicionamento colaborativo entre a NewPedagogies for Deep Learning e o Microsoft Educação. [traduzido para português] Disponível em: http://aka.ms/HybridLearningPaper Acesso em: $07 \mathrm{abr}$. 2021.

IFB. Instituto Federal de Brasília (2014) Plano de Curso Técnico em Informática Integrado a Ensino Médio. Brasília, DF. Disponível em: https://www.ifb.edu.br/index.php/estude-no-ifb?id=8026 Acesso em: 20 mai. 2021.

Moran, J. (2018) Metodologias ativas para uma aprendizagem mais profunda. In: Bacich, L. e Moran, J. (Orgs.) "Metodologias ativas para uma educação inovadora: uma abordagem teórico-prática". Porto Alegre: Penso, 2018. p. 1-25.

Nunes, J. B. C (2015) "Estado da Arte sobre Analítica da Aprendizagem na América Latina", Anais dos Workshops do Congresso Brasileiro de Informática na Educação, p. 1024-1033. DOI: http://dx.doi.org/10.5753/cbie.wcbie.2015.1024. Acesso em: 06 abr. 2021.

Petri, G., Battistella, P. E., Von Wangenheim, C. G., Cassettari, F. T., Hauck, J. C. R. (2016) "Um Quiz Game para a Revisão de Conhecimentos em Gerenciamento de Projetos", Simpósio Brasileiro de Informática na Educação, 23., p. 320-329. DOI: http://dx.doi.org/10.5753/cbie.sbie.2016.320 Acesso em: 30 mar. 2021.

SoLAR. Society for Learning Analytics Research (2021) About SoLAR. What is Learning Analytics? Disponível em: https://www.solaresearch.org/about/what-islearning-analytics/. Acesso em 06 abr. 2021. 